\title{
Shrinking gold nanoparticles: dramatic effect of a cryogenic process on tannic acid/sodium citrate-generated gold nanoparticles
}

\author{
C.Y. Tsai ${ }^{\text {a }}$, D.S. Lee ${ }^{\text {a }}$, Y.H. Tsai ${ }^{\text {a }}$, B. Chan ${ }^{\text {b }}$, T.Y. Luh ${ }^{\text {c }}$, P.J. Chen ${ }^{\text {d }}$, P.H. Chen ${ }^{\text {a,* }}$ \\ ${ }^{a}$ Department of Mechanical Engineering, National Taiwan University, No. 1, Sec. 4, Roosevelt Rd., Taipei 10617 Taiwan, ROC \\ ' Institute of Chemistry, Academia Sinica, Nankang, Taipei 115 Taiwan, ROC \\ ${ }^{\mathrm{c}}$ Department of Chemistry, National Taiwan University, Taipei 10617 Taiwan, ROC \\ ${ }^{\mathrm{d}}$ Hepatitis Research Center, National Taiwan University Hospital, Taipei 10617 Taiwan, ROC
}

Received 21 November 2003; accepted 10 December 2003

\begin{abstract}
Aqueous gold nanoparticles (Au-NPs) without surfactant protection undergo irreversible aggregation upon a cryogenic treatment. However, a cryogenic process on aqueous gold nanoparticle solution containing tannic acid did not lead to nanoparticle aggregation. The tannic acid serves as surfactant to prevent aggregation of gold nanoparticles. In contrast, a reduction in the particle size in this solution containing tannic acid was observed upon the cryogenic treatment with good reproducibility and uniformity.

(C) 2004 Elsevier B.V. All rights reserved.
\end{abstract}

Keywords: Shrinking; Gold nanoparticle; Cryogenic process; Tannic acid; Trisodium citrate

\section{Introduction}

Many nano-scale materials exhibit unique properties that are ideal for applications in science and technology [1-3]. Among them gold nanoparticles (Au-NPs) appear rather promising. Au-NPs can be synthesized chemically as aqueous or organic solutions. In general, organic phase syntheses produce smaller Au-NPs $(<10 \mathrm{~nm})$ than those prepared as aqueous solutions $(>10 \mathrm{~nm})[4-6]$. On the other hand, aqueous phase syntheses are usually superior in their simplicity. The reaction media (water) and the commonly used reagents involved, such as citrate, tannic acid, and ascorbic acid, are also more benign to the environment than organic reaction media. Therefore, the means to alter and possibly control the size of the aqueous Au-NPs is desirable.

Once Au-NPs are formed, it is generally very difficult to change their size with present technologies. It has recently been reported that the particle size of Au-NPs can be reduced by irradiating an aqueous Au-NP solution with a laser beam. Accordingly, the mean particle diameter changed from 37 to $10 \mathrm{~nm}$ [7]. Herein, we report another

\footnotetext{
* Corresponding author. Tel.: +886-2-23670781; fax: +886-223670781/+886-2-23631755

E-mail address: phchen@ntu.edu.tw (P.H. Chen).
}

method for the reduction of Au-NP particle size by means of a cryogenic process.

\section{Results and discussion}

$\mathrm{Au}-\mathrm{NPs}$ were prepared by aqueous phase reduction using either sodium citrate only or both sodium citrate and tannic acid according to literature procedures [11]. The tannic acid can serve as surfactant to prevent aggregation of Au-NPs. The resulting Au-NP solutions were ruby in color, as shown in Figs. 1 and 2. In all cases, the UV-Vis (UV-Vis spectrometer, Hitachi U3310) absorption maxima of these solutions were all in the range $520-525 \mathrm{~nm}$. The sizes of the Au-NPs were found to be in the range 5-28 $\mathrm{nm}$ by high-resolution transmission electronic micrograph (HR-TEM, model: H-7000, Hitachi) using an accelerating voltage of $100 \mathrm{kV}$ with a mean diameter of $17 \mathrm{~nm}$. Cryogenic experiments were performed on an Applied Cryogenics, CP-100 cryocooler under a chamber pressure of $1 \mathrm{~Pa}[8-10]$. Au-NP solutions were cooled from 300 to $77 \mathrm{~K}$ over a period of $12 \mathrm{~h}$ with a temperature rampingdown rate of $0.3 \mathrm{~K} / \mathrm{min}$; this was followed by warming the solutions to $300 \mathrm{~K}$ at a temperature ramping-up rate of 0.3 $\mathrm{K} / \mathrm{min}[12,13]$. 


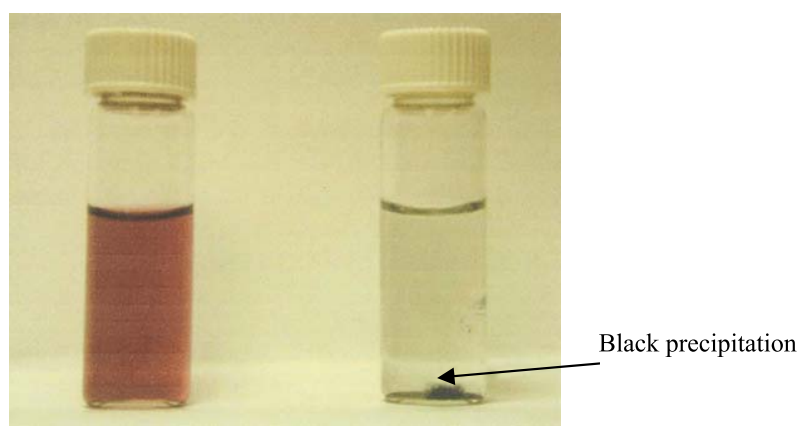

(a)

(b)

Fig. 1. Aqueous Au-NPs solution without tannic acid in (a) ruby color before a cryogenic process; (b) limpid color after a cryogenic process.

For Au-NP solutions prepared with sodium citrate only, the color changed from ruby to limpid after the cryogenic process. As shown in Fig. 1, black precipitates gradually formed, leaving a clear colorless solution. The precipitate was not soluble in deionized water after the temperature of aqueous Au-NP solution temperature is restored back to room temperature. On the other hand, applying the same cryogenic process to Au-NP solution prepared with tannic acid/sodium citrate led to a color change from ruby to brownish yellow, as shown in Fig. 2. In contrast, no precipitation was found in the aqueous Au-NP solution with tannic acid/sodium citrate after the cryogenic process. As shown in Fig. 3, the UV-Vis spectrum of this solution with tannic acid/sodium citrate has a lower absorption maximum at $513 \mathrm{~nm}$ surface plasmon band compared to $523 \mathrm{~nm}$ to that of the original solution. This is consistent with a reduction in particle size from $5-28$ to $2-6 \mathrm{~nm}$ [14], which is confirmed by TEM images, as shown in Figs. 4 and 5, which shows the size distributions of Au-NPs with tannic acid protection before and after the cryogenic process. The mean diameter of downsized Au-NPs is around $4 \mathrm{~nm}$. The phenomenon of this "particle-shrinking" was found to be

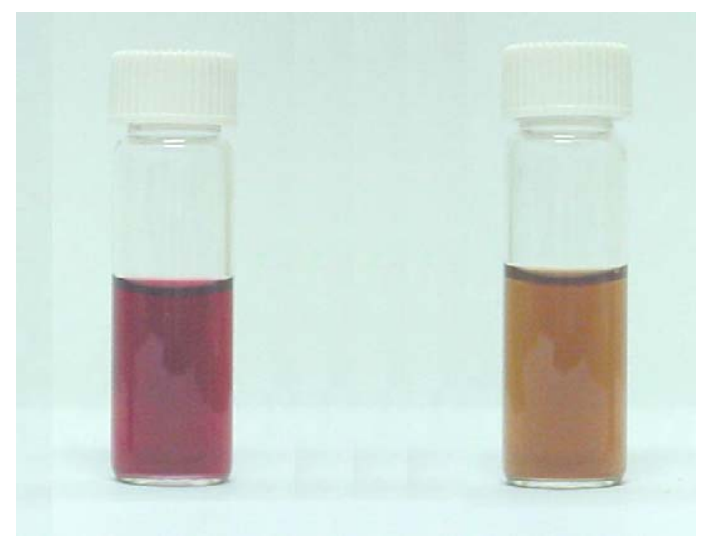

(a)

(b)

Fig. 2. Aqueous Au-NP solution with tannic acid in (a) ruby color before a cryogenic process; (b) brownish yellow color after a cryogenic processing.

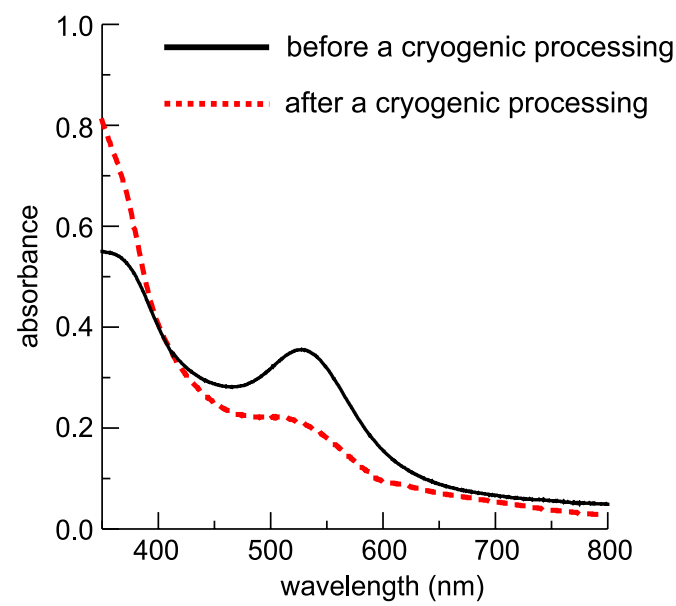

Fig. 3. UV-Vis spectra of aqueous Au-NP solution with tannic acid as surfactant.

reproducible over three independent runs on three independent samples. In addition, the variation in particle's diameter is less than $2 \mathrm{~nm}$ after the Au-NP aqueous with tannic acid/ sodium citrate is treated with the cryogenic process.

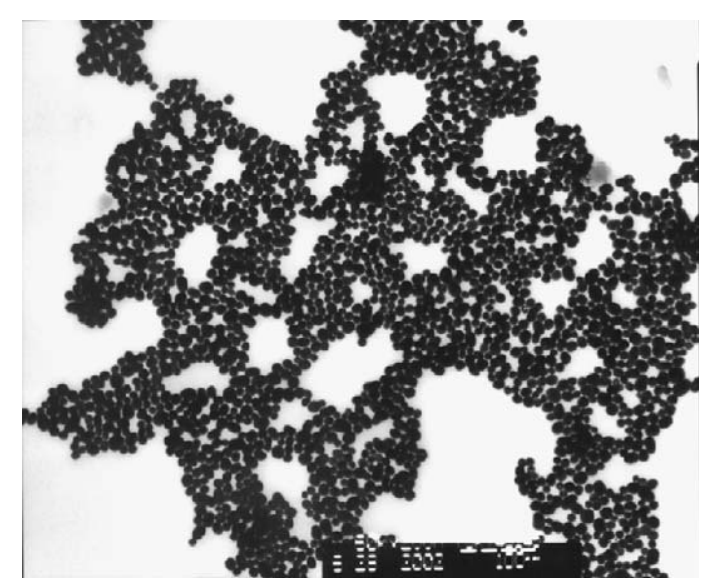

(a)

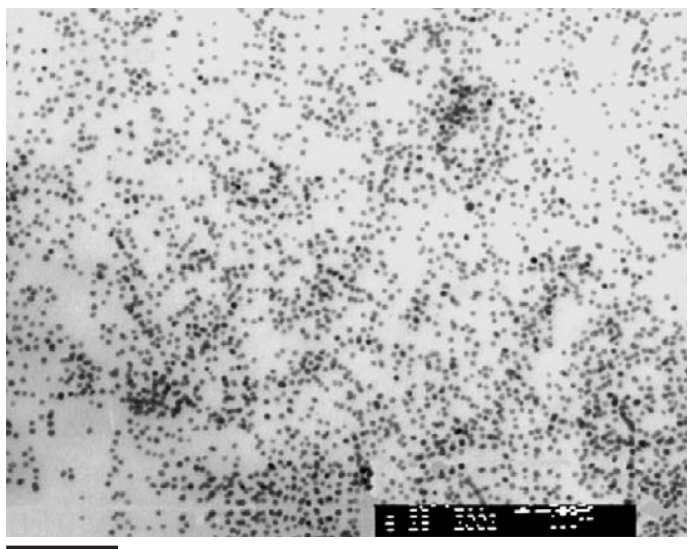

$100 \mathrm{~nm}$

(b)

Fig. 4. TEM image of Au-NPs with tannic acid as surfactant at a magnification of 200,000: (a) before a cryogenic processing; (b) after a cryogenic processing. 


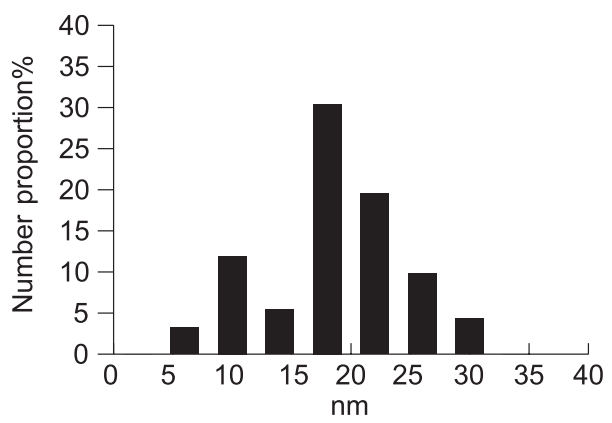

(a)

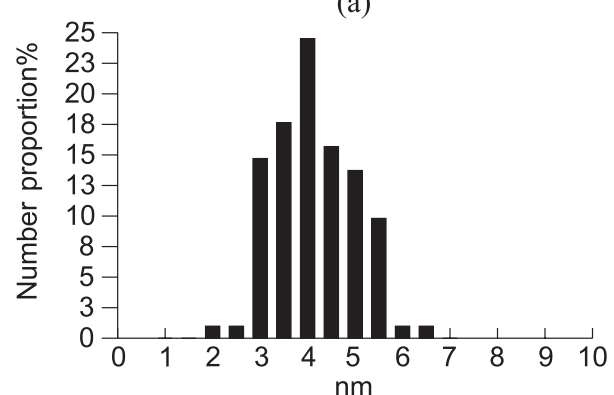

(b)

Fig. 5. Size distribution of Au-NPs with tannic acid obtained from TEM images: (a) before a cryogenic processing; (b) after a cryogenic processing.

Figs. 6 and 7 show both bright field and dark field HRTEM micrographs and the selected-area electron beam diffraction (SAED) pattern of gold nanoparticles before and after the cryogenic process, respectively. The SAED pattern of gold nanoparticles can be used to characterize the structure constitution of Au-NPs before and after the cryogenic process. The selected area, marked by a circle in Figs. $6 \mathrm{~A}$ and $7 \mathrm{~A}$, for electron beam diffraction pattern measurement is around $100 \mathrm{~nm}^{2}$. The Debye-Scherrer rings in SAED patterns, shown in Figs. 6C and $7 \mathrm{C}$, indicate the gold nanoparticles before and after the cryogenic process have a crystalline structure and not an amorphous structure $[15,16]$.

The gold nanoparticles before the cryogenic process, shown in Fig. 6A and B, have an average diameter of 17 $\mathrm{nm}$. Every bright spot in dark field HR-TEM microgarph shows a single gold nanoparticle of which the formation is not due to the aggregation of smaller nanoparticles. As observed from Fig. 6C, four Debye-Scherrer rings corresponding to (111), (200), (220), and (311) planes of an fcc crystalline lattice, strong in both (111) and (200) planes but weak in (220) and (311) planes [15,16]. This structural characteristic shows that the gold nanoparticles before the cryogenic process have anisotropic crystalline structure. The particle size of gold nanoparticles after the cryogenic process is around $4 \mathrm{~nm}$, as shown in Fig. 7A and B. Two Debye-Scherrer rings in SAED pattern, shown in Fig. 7C, are corresponding to (111) and (200) planes of an fcc lattice. In addition, the bright spots in Fig. 7B are smaller in size than those in Fig. 6B. This indicates that the particle size of every single gold nanoparticle, not an aggregation of smaller nanoparticles, before the cryogenic
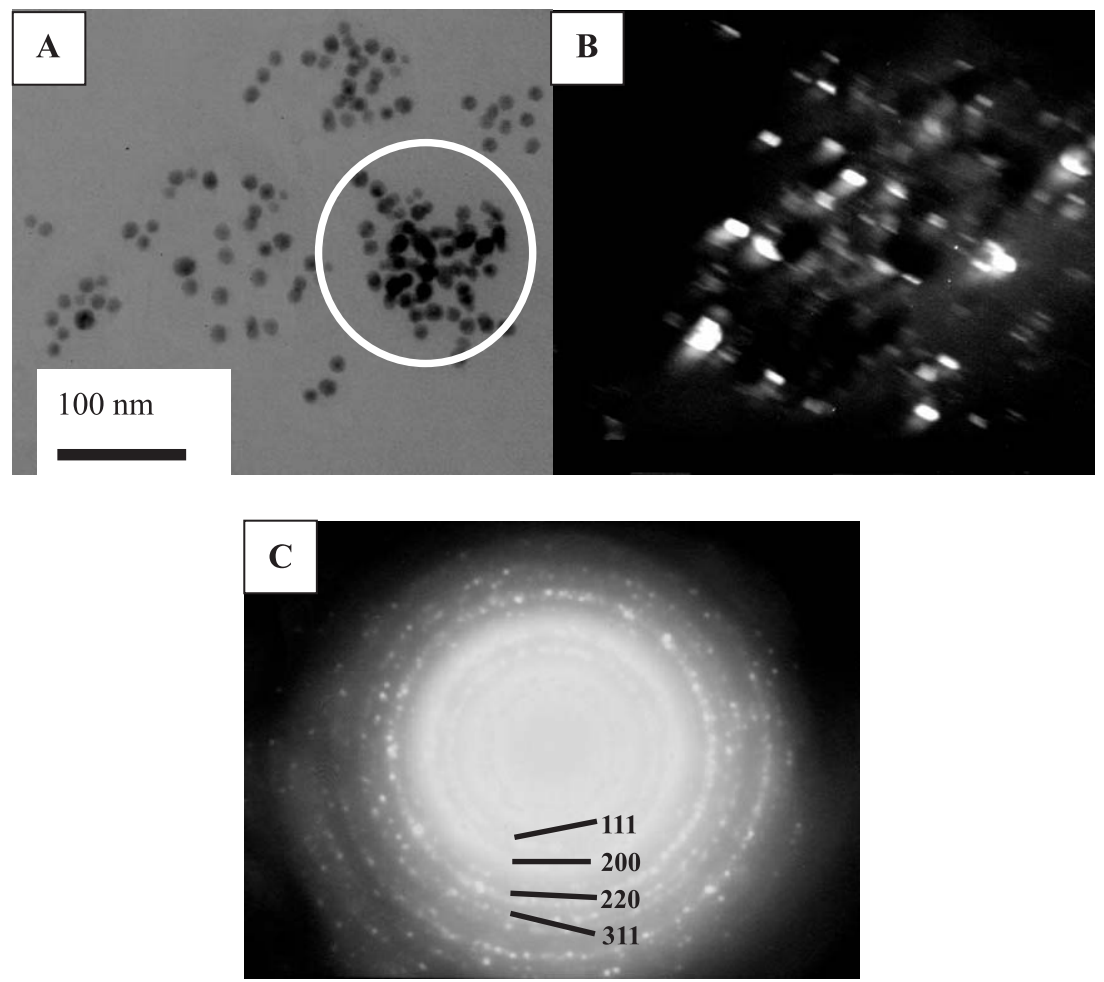

Fig. 6. TEM micrographs of Au-NPs before a cryogenic processing: bright field image (A), dark field image (B), and SAED pattern (C). 

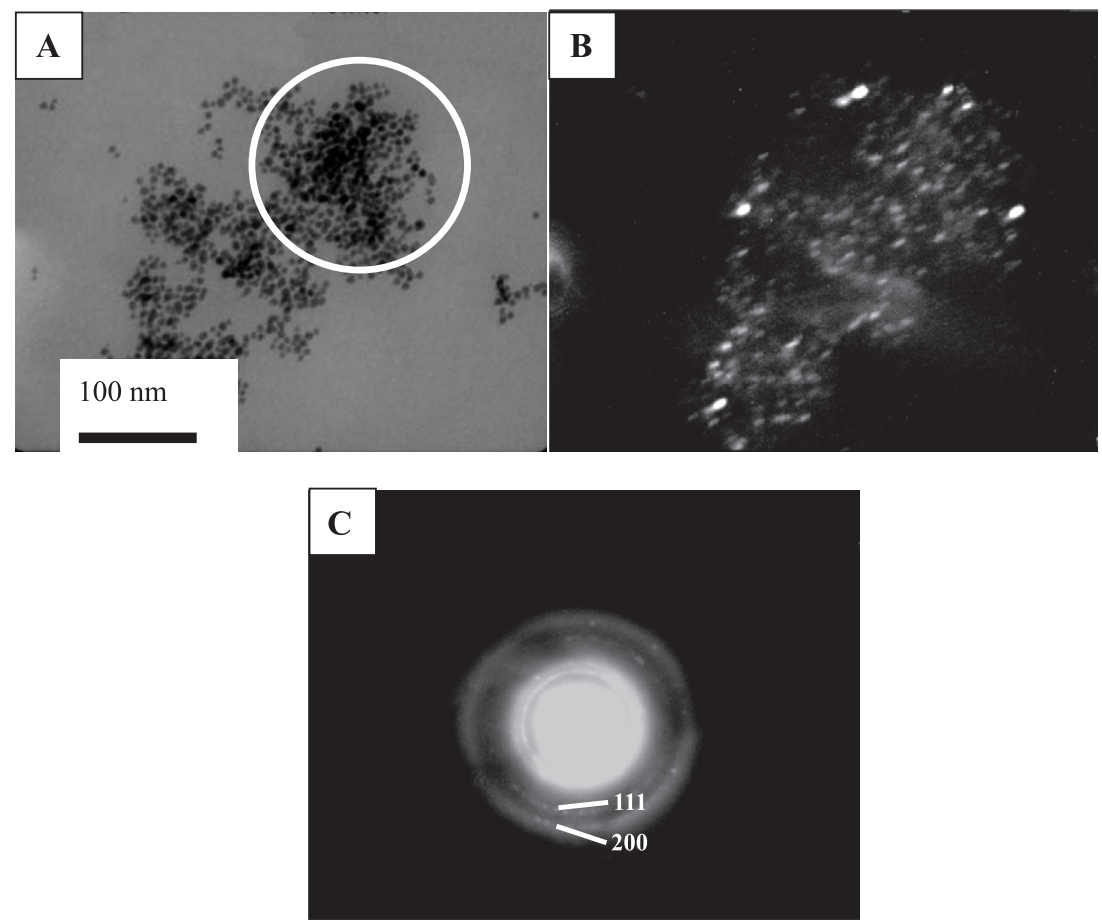

Fig. 7. TEM micrographs of Au-NPs after a cryogenic processing: bright field image (A), dark field image (B), and SAED pattern (C).

process is actually larger than that after the cryogenic process.

\section{Conclusion}

Aqueous Au-NPs solution prepared from either sodium citrate only or both sodium citrate and tannic acid undergo irreversible aggregation upon a cryogenic treatment. In contrast, the same cryogenic treatment on tannic acid/ sodium citrate-generated Au-NPs solution does not lead to the particle's aggregation but a reduction in nanoparticle size from a mean diameter of 17 to $4 \mathrm{~nm}$. In addition, the downsized Au-NPs have a uniform size with a variation of 2 $\mathrm{nm}$ in particle diameter.

\section{Acknowledgements}

We gratefully acknowledge the financial support by the National Science Council, No. NSC-92-2212-E-002-050.

\section{References}

[1] U. Kreibig, M. Vollmer, Optical Properties of Metal Clusters, Springer, Berlin, 1995.
[2] A. Takami, H. Yamada, K. Nakano, S. Koda, Jpn. J. Appl. Phys. 35 (1996) L781.

[3] H. Kurita, A. Takami, S. Koda, Appl. Phys. Lett. 72 (1998) 789.

[4] M. Brust, M. Walker, D. Bethel, D.J. Schiffrin, R. Whyman, J. Chem. Soc. Chem. Commun. (1994) 801.

[5] V.F. Puntes, K.M. Krishnan, A.P. Alivisatos, Science 291 (2001) 2115.

[6] L. Guo, Q. Huang, X.Y. Li, S. Yan, Phys. Chem. Chem. Phys. 3 (2001) 1661

[7] A. Takami, H. Kurita, S. Koda, Laser-induced size reduction of noble metal particles, J. Phys. Chem. B 103 (1999) 1226.

[8] J.R. Hull, Energy Conversion Engineering Conference, IECEC-89 1 (1989) 459.

[9] R.L. Patterson, A. Hammoud, J.E. Dickman, S. Gerber, M. Elbuluk, E. Overton, J. Phys. IV 12 (2002) 207.

[10] J. Hagen, L.D. Socaciu, M. Elijazyfer, U. Heiz, T.M. Bernhardt, L. Woüste, Phys. Chem. Chem. Phys. 4 (2002) 1707.

[11] M.A. Hayat, Colloidal Gold: Principles, Methods, and Applications, vol. 1, Academic Press, San Diego, 1989, chap. 2.

[12] R.F. Barron, Cryogenic Systems, Oxford University Press, London, 1985.

[13] R.F. Barron, A study of the effects of cryogenic treatment on tool steel properties, Louisiana Technical University Report, August 30, 1973.

[14] S. Link, M.A. El-Sayed, J. Phys. Chem. B 103 (1999) 8410.

[15] J.W. Edington, Practical Electron Microscopy in Material Science, Van Nostrand Rheinhold, New York, 1976.

[16] B.D. Cullity, Element of X-Ray Diffraction, Addision-Wesley, Reading, MA, 1978. 